\title{
Conception et mobilisation d'une boussole pédagogique dans le cadre d'un dispositif de formation par alternance
}

Analyse du développement professionnel d'une enseignante stagiaire en EPS

Conception and operated a device of training by alternation to help pre-service teachers to articulate theorical knowledge and empirical knowledge: Analysis the professional development of a pre-service teacher of physical education.

Jérôme Guérin, Gilles Kermarrec et Jacky Péoc'h

\section{OpenEdition}

Édition électronique

URL : http://journals.openedition.org/educationdidactique/783

DOI : $10.4000 /$ educationdidactique.783

ISBN : 978-2-7535-1624-3

ISSN : 2111-4838

\section{Éditeur}

Presses universitaires de Rennes

\section{Édition imprimée}

Date de publication : 1 octobre 2010

Pagination : $21-40$

ISBN : 978-2-7535-1300-6

ISSN : 1956-3485

\section{Référence électronique}

Jérôme Guérin, Gilles Kermarrec et Jacky Péoc'h, « Conception et mobilisation d'une boussole pédagogique dans le cadre d'un dispositif de formation par alternance », Éducation et didactique [En ligne], 4-2 | 2010, mis en ligne le 01 avril 2012, consulté le 09 décembre 2020. URL : http:// journals.openedition.org/educationdidactique/783 ; DOI : https://doi.org/10.4000/ educationdidactique. 783 


\title{
CONCEPTION ET MOBILISATION D'UNE BOUSSOLE PÉDAGOGIQUE DANS LE CADRE D'UN DISPOSITIF DE FORMATION PAR ALTERNANCE :
}

\author{
ANALYSE du DÉVELOPPEMENT PROFESSIONNEL D'UNE \\ ENSEIGNANTE STAGIAIRE EN EPS
}

\author{
Jérôme Guérin, Gilles Kermarrec E Jacky Péoc’h \\ (IUFM de Bretagne - UBO)
}

\begin{abstract}
Résumé : Cet article décrit comment un dispositif de formation par alternance veut répondre à la demande d'aide d'enseignants stagiaires d'éducation physique et sportive (EPS) souhaitant enseigner en référence à une conception socioconstructiviste de l'apprentissage. La recherche/intervention est menée en mobilisant les concepts et méthodes proposés par l'ergonomie cognitive pour l'analyse de l'activité humaine. Nos résultats présentent en trois temps le processus du développement professionnel d'une professeur stagiaire enseignant la gymnastique à des élèves de troisième. Dans une première partie, nous montrons que les connaissances théoriques se sont progressivement stabilisées, donnant aux préparations de leçon plus de cohérence et leur conférant un réel statut de ressources pour saisir des opportunités en classe. Ensuite nous décrivons l'évolution des préoccupations et des connaissances qui organisent l'activité de l'enseignante stagiaire en classe. Enfin la dernière partie des résultats montre que la dimension collective des moments partagés entre formateurs et enseignants stagiaires est une dimension de la formation précieuse et favorable au processus de développement professionnel. La discussion porte sur la circularité des connaissances comme processus du développement professionnel, l'effet transformateur des entretiens individuels et des échanges collectifs, la migration des connaissances comme saisies d'opportunités et l'évolution des préoccupations co-construites lors des séquences de formation.
\end{abstract}

Mots clés : conception socioconstructiviste, enseignant-stagiaire, développement, ergonomie cognitive, dispositif de formation

\section{Jérôme Guérin E al}

\section{Introduction}

À partir d'options théoriques et méthodologiques différentes, les chercheurs ont souvent analysé et décrit l'activité des stagiaires lors de la formation initiale des enseignants stagiaires du premier et du second degré proposée au sein des IUFM (Institut Universitaire de Formation des Maîtres) comme un produit du dispositif de formation professionnelle. Cependant, ces travaux de recherche se sont souvent opposés selon qu'ils s'intéressent aux compétences des enseignants à penser et planifier leur pratique, ou qu'ils privilégient l'étude des compétences à agir, intervenir en situation réelle.

Depuis quelques années, dans le champ des sciences de l'éducation, c'est l'analyse de l'activité des enseignants stagiaires au sein des dispositifs de formation qui génère le plus grand nombre de productions. L'activité des enseignants stagiaires a ainsi été analysée lors des entretiens de conseil pédagogique post-leçon (Anderson, 1992, Chaliès
\& Durand, 2000), lors de la rédaction des mémoires professionnels (Cros, 1999; Gonnin-Bolo, 2002) ou lors d'ateliers d'analyse de pratique (Altet, 2000; Méard, 2004). Si ces travaux ont permis d'identifier les savoirs et les compétences pour exercer le métier d'enseignant (Tardif et Lessard, 1999), ils ne disent rien du processus de formation et de son impact sur le développement du pouvoir d'action en classe des enseignants stagiaires. C'est pourquoi des travaux ont entrepris d'évaluer l'impact sur le développement professionnel des dispositifs alternant les moments de formation à l'IUFM et l'activité des enseignants stagiaires en classe. Sans prétendre réaliser une revue de littérature sur cette question, nous avons choisi ici de présenter les approches théoriques et méthodologiques qui ont nourri la conception de notre propre travail.

La première approche est développée par des membres de l'équipe Développement de l'Activité Travail et Identité des Enseignants en Formation (DATIEF) de l'Unité Mixte de Recherche ADEF 
(Bertone, Chaliès, Clarke, Méard, 2006) et associe la clinique de l'activité en psychologie du travail (Clot, 1999) et la philosophie de l'action (Wittgenstein, 2004). Les travaux menés dans cette perspective portent sur l'analyse des situations de formation initiale des enseignants et se proposent d'identifier et de caractériser le développement de l'activité professionnelle au cours des échanges entre stagiaire (novice) et tuteur (expert) pour répondre à la question suivante: comment les enseignants novices élaborent, en interaction avec des formateurs universitaires ou de terrain, des règles et des techniques professionnelles (Méard et Bruno, 2009). L'enjeu est ici de comprendre comment les règles apprises en dehors du contexte où elles devront s'appliquer peuvent être utilisées pour agir et penser en classe. Cette approche pose comme centrale la question des conditions de la migration des apprentissages issus des expériences de formation vers la classe. C'est dans cette perspective que Chaliès, Bertone, \& Flavier (2005) ont entrepris d'évaluer les dispositifs de formation fondés sur les pratiques réflexives afin de préciser les circonstances dans lesquelles le travail entre le stagiaire et le tuteur était une occasion de développement professionnel.

La question de l'efficacité des dispositifs de formation est aussi une préoccupation partagée par Amigues, Faita et Saujat, (2004) qui ont choisi d'exploiter les principes de la théorie socioculturelle de l'apprentissage et du développement de Vygotski (1997). Ces auteurs défendent l'idée que la collaboration entre chercheur (s) et acteur(s) doit mettre en discussion la façon de « faire le métier » des seconds pour comprendre et transformer leur activité professionnelle. Cette collaboration repose notamment sur l'instauration, entre l'analyste du travail et les acteurs, d'un milieu de co-analyse et de construction des faits (Daniellou, 1996). Plus précisément ce sont des méthodes indirectes telles que l'instruction au sosie ou l'autoconfrontation simple et croisée (Clot, Faita, Fernandez, \& Scheller, 2001) qui sont exploitées pour mettre à distance et en mouvement l'activité réalisée afin d'ouvrir de nouveaux possibles d'action en classe. C'est à cette condition, c'est-à-dire en continuant dans un cadre dialogique à « distordre » l'expérience en classe que les enseignants guidés par des formateurs trouveraient collectivement des règles professionnelles pour surmonter l'obstacle rencontré (Saujat, 2005). Ce qui nous a semblé intéressant dans cette orientation de recherche en ergonomie et psychologie du travail, c'est non seulement de vouloir comprendre l'activité réalisée des enseignants stagiaires mais aussi ce qui la contrarie et lui conteste son développement, les possibilités non réalisées, écartées, inhibées ou empêchées (Clot, 1999).

Selon les principes et outils du cadre théorique et méthodologique du cours d'action (Theureau 2004, 2006) les travaux menés au sein du laboratoire Processus d'Action des Enseignants Déterminants et Impacts (PAEDI) de l'IUFM d'Auvergne dirigé par Ria (2006) se sont donnés comme axe de recherche la caractérisation des modalités de développement de l'activité professionnelle d'enseignants stagiaires en EPS au gré des contextes de classe et de formation. Serres, Ria, \& Adé (2004) ont ainsi retracé de façon synthétique et historique les transformations complexes et conjointes des composantes cognitives, émotionnelles et perceptives de l'activité des enseignants stagiaires lors de la participation à différents contextes de classe et de formation. À travers cette étude, les auteurs ont ainsi montré que la mobilisation et la construction de connaissances dans et par l'action étaient une catégorie d'analyse pertinente pour rendre compte du développement professionnel. Les critères qui ont été retenus pour identifier l'impact de l'action de formation étaient les suivants: (1) la validation ou invalidation par l'enseignant stagiaire de l'énoncé du formateur lors de l'entretien de formation; (2) le prolongement ou non sous la forme d'une activité réflexive au cours de la préparation de la leçon suivante, (3) la validation ou non de son action au cours de la leçon suivante. Grâce à ces critères, Serres et al. (2004) ont caractérisé trois modalités de développement qui montrent que les connaissances validées par l'enseignant stagiaire en formation ne sont pas toutes validées lors de la leçon. Dans le prolongement de cette étude Ria, Leblanc, Serres, \& Durand (2006) ont précisé que l'impact de la formation et notamment des connaissances validées en formation par les stagiaires était lié à la capacité des formateurs à identifier et répondre aux préoccupations et attentes des stagiaires.

Malgré des références théoriques et méthodologiques différentes, ces approches du développement professionnel ont en commun à la fois de privilégier une entrée par l'expérience professionnelle ${ }^{1}$ plutôt qu'une entrée par les savoirs disciplinaires et d'exploiter des méthodes issues de l'analyse du travail. Ces approches reconnaissent toutes que c'est dans 
l'articulation des différentes expériences de formation et de pratique qu'il faut chercher le développement de l'enseignant débutant. C'est pourquoi, à différents niveaux, elles tendent d'évaluer l'impact de l'action des formateurs et de la formation sur l'amélioration de la qualité des pratiques réelles d'intervention en classe.

$\mathrm{Au}$ niveau théorique, ces études se reconnaissent également dans une approche d'ergonomie cognitive qui envisage la formation « comme une construction empirique et scientifique, évolutive et adaptative articulée à une analyse de l'activité » qui s'organise comme "une démarche itérative alternant quatre phases: a) analyse de l'activité située au travail et en formation, b) conception « orientéeactivité » de situations de formation, c) analyse de l'activité des acteurs dans ces nouveaux espaces de formation, d) retour sur la conception du dispositif (p. 59, Leblanc, Ria, Dieumegard; Serres, \& Durand, 2008) ». Tout comme ces auteurs, nous définissons l'activité humaine en référence à six présupposés épistémologiques. (1) L'activité est considérée comme une totalité indissociable: l'émotion, l'action, les communications, le jugement, la perception forment un tout. (2) Elle est située car liée à la situation matérielle, sociale et culturelle au sein de laquelle elle prend forme. (3) Elle est dynamique, évolutive et donne lieu à la construction et à la validation/invalidation de connaissances (4) L'activité humaine est auto-organisée et l'acteur entretient une relation asymétrique avec son contexte. Il ne prend en considération que les éléments du contexte qui sont significatifs par rapport à ses intentions à l'instant t. (5) L'activité d'un acteur présente des traits communs avec d'autres acteurs appartenant à une même communauté de pratique (Wenger, 1998). Les autres sont considérés comme une ressource pour apprendre et satisfaire les normes et les valeurs de la communauté. (6) Enfin l'activité donne lieu à expérience dans le sens où l'acteur est un être vivant autonome engagé dans une pratique qu'il peut, à un certain niveau de conscience, expliciter à un observateur interlocuteur.

Ces présupposés invitent à considérer les situations d'enseignement et de formation comme autonomes et articulées, c'est-à-dire faites d'interactions permanentes entre action, conception de dispositifs et analyse de pratiques.
Au regard des travaux déjà réalisés et de la difficulté à pronostiquer l'effet d'un dispositif de formation par alternance sur le développement professionnel de l'activité en classe, on peut penser que des dispositifs innovants sont encore à créer pour faciliter et accompagner le développement de compétences professionnelles. Pour compléter les présupposés relatifs à une approche "orientée activité * (Barbier et Durand, 2003), nous ajoutons deux principes technologiques qui optimisent l'efficacité du dispositif d'aide au développement professionnel. Le premier, emprunté à Méard et Bruno (2009), consiste à affirmer que la compétence professionnelle de l'enseignant s'acquiert lorsque le stagiaire exerce mais également lorsqu'il y a une identification de connaissances utiles et un pointage des règles de métier par des formateurs. Le second principe consiste à donner le primat aux préoccupations, aux attentes des stagiaires, y compris à ce qu'ils n'ont pas osé faire lors de leur intervention en classe pour poursuivre ou maintenir le cours des transactions entre formateurs et stagiaires. En effet, compte tenu du postulat d'autonomie de l'enseignant stagiaire, les outils, les conseils du formateur ne pourront être considérés comme des ressources pour maintenir ou adapter son organisation propre que si ceux-ci répondent aussi à ses préoccupations et ses attentes hic et nunc. Ces principes sont fondés d'une part par les connaissances relatives au mécanisme du développement défini par Clot (2007) et d'autre part sur l'affirmation partagée par les enseignants novices que l'expérience de l'intervention en classe est le principal élément de formation professionnelle.

C'est à partir de cette approche ergonomique de l'activité d'enseignement (Theureau, 2006) que nous avons envisagé de mettre en œuvre et d'analyser l'impact d'un dispositif de formation par alternance conçu à partir d'une demande d'aide d'enseignants stagiaires d'éducation physique et sportive (EPS). Outre la visée transformative de l'activité en classe des enseignants stagiaires, il s'est agi aussi, lors de cette étude, de rendre compte des liens entre les connaissances construites en dehors de la classe et celles effectivement mobilisées en classe.

L'objectif de cet article est donc de présenter un dispositif construit à partir d'une demande d'enseignants stagiaires souhaitant enseigner l'éducation physique selon une conception socio-constructiviste 
de l'apprentissage. Plus précisément, nous décrivons comment l'activité de formation au sein du dispositif a déclenché des processus d'apprentissage/développement favorables au déploiement d'une activité plus confortable car cohérente, et dont l'impact effectif se rapproche de celui attendu.

Nous nous référons à la définition de Durand (2008) pour qui "l'apprentissage est l'acquisition par les individus et les collectifs qu'ils constituent, de modes d'action nouveaux pour eux en relation ou non avec un projet intentionnel de transmission de ces modes d'action par une instance de formation; le développement est la transformation du répertoire d'actions des individus et les collectifs qu'ils constituent, en relation ou non avec un projet intentionnel d'accompagnement par une instance de formation Durand, 2008 p. 2)". Il semble important de souligner ici que les postulats relatifs à l'activité humaine donnent aux connaissances théoriques relatives à l'approche socio-constructiviste de l'apprentissage un statut de ressource, d'aide pour intervenir, et non de connaissances à appliquer. Dans l'environnement incertain et dynamique de la classe, il s'agira donc d'aider les enseignants stagiaires à reconnaître et saisir les opportunités pour mobiliser les connaissances relatives à cette conception de l'apprentissage.

Ce compte rendu de recherche / intervention ${ }^{2}$ n'est qu'une partie d'un programme de recherche plus ambitieux qui vise à apporter des éléments de réponse à quelques questions récurrentes. Comment conception et action s'alimentent-elles réciproquement? Comment les connaissances produites et mobilisées dans et par l'activité collective de formation migrentelles dans l'espace de la classe? Celles-ci entrentelles en concurrence ou non avec des connaissances construites dans et par l'action en classe? Comment les connaissances font-elles l'action tout en émergeant mais aussi comment fondent-elles une conception, un réseau, un système par l'activité collective?

\section{Méthode}

\section{Contexte et participants à l'étude}

Notre recherche a été menée à l'IUFM de Bretagne, école interne de l'Université de Bretagne Occidentale (UBO) avec trois enseignants - stagiaires ${ }^{3}$ en éducation physique et sportive (EPS). Tout au long de l'année scolaire, ces derniers enseignaient à deux classes en situation de responsabilité et participaient une ou deux fois par semaine à des actions de formation à l'IUFM. C'est en marge des dispositifs de formations institutionnels que les enseignants stagiaires ont fait la demande à deux des trois enseignants chercheurs co-auteurs de l'article de les aider à construire et mettre en œuvre un enseignement conforme à une conception socio-constructiviste de l'apprentissage ${ }^{4}$. Cette dernière, dans le cadre de l'éducation physique, recouvre un ensemble large et hétérogène de pratiques et de discours mettant en avant l'idée d'un apprentissage en action, fondé essentiellement sur les interactions entre les élèves et sur des moments de mise à distance de l'action. Pour ces stagiaires l'usage et l'intégration de connaissances théoriques dans la pratique de leur travail quotidien fondée sur le «learning by doing " de John Dewey se présentaient à la fois comme une préoccupation, qu'elle soit d'ordre éthique (ou idéologique au sens de Mialaret 1996) ou pragmatique (visée d'efficacité), mais aussi comme une difficulté récurrente.

\section{Dispositif de formation}

À la suite de cette demande, les enseignants chercheurs et les stagiaires ont convenu d'un travail coopératif délimité par un contrat de collaboration écrit définissant les modalités de participation de chacun ${ }^{5}$ et les modalités d'usage des données d'enregistrement audio et vidéo en dehors du cadre de la formation. L'instauration du cadre de collaboration avec les enseignants stagiaires s'est présentée pour les enseignants chercheurs comme une opportunité pour tester l'efficacité d'un dispositif par alternance dépassant l'alternative entre une formation centrée sur l'analyse de l'activité réelle mais sans connaissances théoriques ressources (modèle inductif), et une formation proposant des curriculums structurés par des connaissances scientifiques (modèle déductif), mais sans prise en compte de l'activité réelle en classe (Durand, de Saint Georges, \& Meuwly-Bonte, 2006).

Dans cette perspective le dispositif de formation a donc été composé de trois sessions de travail qui se sont déroulées selon un fonctionnement itératif scandé lui-même en quatre temps. Ce dispositif a été précédé d'une phase initiale consacrée à l'explicitation de la demande et des attentes des stagiaires. 
Cette demande a été formulée sans perdre de vue les exigences d'efficacité (les élèves doivent réaliser des progrès tangibles), les exigences de confort (les stagiaires doivent voir préservée leur intégrité morale, leur capacité à être disponibles, attentifs...), les exigences institutionnelles (liées aux programmes). Ce qui préoccupait à ce moment nos stagiaires était de construire une cohérence entre ce qui demeurait pour eux une "idéologie » (Mialaret, 1996) constructiviste et leur activité réelle de professeur. Cette première phase a en outre été dédiée à la formalisation du dispositif notamment à l'explicitation du rôle occupé par les enseignants chercheurs.

Pour chacune des trois sessions qui ont suivi cette phase initiale de négociation, la formation s'est organisée autour d'un rituel identique, indexé sur les dimensions que nous considérons typiques de l'activité professionnelle, et composé de quatre temps: la préparation de leçon, la leçon vécue, l'entretien d'autoconfrontation et la séquence d'analyse collective. Lors de chaque temps, des traces de l'activité des enseignants stagiaires ont été recueillies.

\section{$1^{\circ}$ temps: la préparation de la leçon}

La planification pour l'action afin d'interroger et "faire vivre» les concepts constructivistes: concrètement et pour éviter l'écueil d'une démarche déductive, il s'est agi dans ces moments d'envisager l'activité professionnelle (tant celle de l'enseignant que, en miroir, celle des élèves) à partir de ces concepts (action, réflexion, interaction, autonomie, processus, introspection...) et de garder les traces de ces réflexions sous forme de fiche de préparation. Celles-ci devant évoluer dans leur conception (forme linéaire, systémique...), dans leur fonction (cadrage, ouverture d'opportunités...), dans leur contenu (place des artefacts: matériel, fiches d'observation, durées...) ont eu alors deux fonctions: la première celle d'organiser la leçon à venir et la seconde celle de servir de trace pour témoigner du développement professionnel des stagiaires. Lors de ce temps collectif les enseignants chercheurs occupaient une posture de formateur visant à aider les stagiaires à expliciter leurs préoccupations et attentes relatives à leur action en classe et celles des élèves. Les échanges provoqués lors de cette phase collective ont été intégralement enregistrés à l'aide d'un petit enregistreur numérique audio. Enfin, des notes ethnographiques ont été prises par les enseignants stagiaires. Ils y ont relaté leur ressenti, la manière dont ils ont vécu le temps de formation.

\section{$2^{\circ}$ temps: la leçon}

Lors de ce temps, le stagiaire est sans doute relativement déchargé de la multiplicité des dilemmes qui pourraient envahir son activité dans la mesure où il connaît les éléments ciblés qui seront analysés par ses pairs et les enseignants-chercheurs. Du point de vue du confort (estime de soi, repère par rapport aux normes expertes, contrôle de la classe...) même si l'imprévu plane toujours sur la leçon, nous avons fait l'hypothèse que le stagiaire aurait préalablement hiérarchisé une échelle de préoccupations se focalisant non plus sur la réussite de la leçon mais sur son développement professionnel dont les échéances auront été négociées. En classe, certaines séquences de l'activité de l'enseignant stagiaire et des élèves ont été intégralement enregistrées à l'aide de deux caméras numériques. Ces séquences ont été préalablement délimitées en fonction des éléments sur lesquels enseignants-chercheurs et enseignants stagiaires souhaitaient se focaliser.

\section{$3^{\circ}$ temps: entretien d'autoconfrontation}

Le troisième temps était consacré à un entretien d'autoconfrontation individuel conduit par un enseignant-chercheur au cours duquel l'enseignant stagiaire était invité, à partir des traces audiovisuelles de son activité en classe, à expliciter la signification de son expérience vécue (Theureau, 2004). Au cours de cet entretien, il était demandé au stagiaire de suspendre toute activité évaluative. La fonction de l'enseignant chercheur était alors d'accompagner le stagiaire dans la description précise de ses attentes, et dans la mise à distance de ses focalisations, de ses émotions, des dilemmes rencontrés etc. Autrement dit, ici il s'agissait de suspendre la posture de formateur qui explique et prescrit des règles du métier au profit de celle du chercheur qui tente, dans le cadre qu'il s'est imposé, d'accéder à l'expérience du stagiaire, notamment à ses préoccupations hic et nunc. Les entretiens ont été intégralement filmés à l'aide d'une caméra numérique afin d'enregistrer simulta- 
nément l'écran du moniteur de télévision sur lequel étaient diffusées les traces de l'activité en classe et les verbalisations provoquées de l'enseignant stagiaire.

$4^{\circ}$ temps: séquence d'analyse collective

Cet entretien d'autoconfrontation de premier niveau (Theureau, 2004) était suivi d'une séquence collective consacrée à des échanges regroupant l'ensemble des stagiaires et les enseignants-chercheurs. Lors du quatrième temps et pour chacune des trois sessions, les échanges ont porté sur la recherche des cohérences, des écarts, voire des incohérences, par rapport aux prévisions, à la planification. À partir des traces audiovisuelles de l'activité de l'enseignant stagiaire, les pairs pouvaient intervenir en sollicitant leur collègue à partir de leurs propres niveaux de développement, de préoccupations. Ces interactions à multiples niveaux, stagiaires/enseignantschercheurs et stagiaires/stagiaires avaient pour vocation à atténuer les effets de conformités ${ }^{6}$ qui biaisent les rapports novice/expert dans la formation à l'IUFM. Ce qui est demeuré essentiel dans la modalité d'intervention choisie, c'était de mettre les stagiaires en situation réellement professionnelle, c'est-à-dire de les inviter à partager et négocier entre pairs, à s'engager dans des choix conceptuels, à valider/invalider ceux-ci au regard d'une approche critique et méthodique de leur activité réelle et de celle de leurs élèves. Cette activité collective a été ponctuée par des demandes de conseils et d'énonciation de règles du métier de la part des stagiaires en direction des enseignants-chercheurs. Enfin, par isomorphisme, les enseignants stagiaires ont aussi été amenés à interroger, juger, caractériser le dispositif dans lequel ils étaient inscrits. Les échanges ont été intégralement enregistrés à l'aide d'un dictaphone numérique. Comme lors du temps 1, les stagiaires ont consigné dans un carnet la manière dont ils avaient vécu cette expérience et ce qu'ils en retenaient.

Le parti pris sur lequel s'est adossé ce dispositif a surtout été celui de penser qu'il pourrait, par un phénomène de circularité, assurer la migration des connaissances d'un contexte à l'autre: la connaissance dans son système idéologique (accès à l'intelligibilité), la connaissance comme outil de planification (accès à l'ingénierie), la connaissance comme repère pour l'ac- tion hic et nunc (accès à l'épaisseur phénoménologique du réel). Il nous a semblé que, par des phénomènes de rappel, de typification, de pointage... ces connaissances pouvaient, dans un mouvement dialectique, migrer de contextes génériques $^{7}$ à des contextes circonstanciés ${ }^{8}$ (Méard \& Bruno, 2009) et inversement.

\section{Analyse des données}

Le traitement des données a été réalisé en mobilisant le cadre sémiologique du cours d'action (Theureau, 2004). Celui-ci intègre les présupposés relatifs à l'activité humaine définis ci-dessus et permet une description du processus de développement en donnant le primat au point de vue de l'acteur sur la description extrinsèque des contingences dans lesquelles ils se réalisent.

Les données ont été traitées en trois étapes: i) description des comportements et la transcription des verbalisations des enseignants stagiaires tant en classe qu'au cours de l'interaction de formation; ii) identification et nomination des composantes de leur cours d'action; iii) identification des séquences du cours d'action.

Descriptions des comportements et transcription des verbalisations

La première étape a consisté à transcrire les données d'enregistrement et les verbalisations provoquées. À chaque fois que cela a été possible, nous avons construit des protocoles à deux volets, plaçant en vis à vis une description des comportements et communications des élèves et de l'enseignant stagiaire en classe et les verbatim des verbalisations issues des entretiens d'autoconfrontation. Ce principe de mise en forme a aussi été réalisé pour les données d'enregistrement des échanges collectifs et le contexte de leur émergence.

Identification et nomination des composantes des signes des cours d'action

La constitution des protocoles a ensuite été exploitée pour documenter pas à pas les éléments de signification accompagnant l'activité de l'enseignant 
stagiaire lors des différents temps du dispositif. Plus précisément nous avons documenté quatre composantes de l'activité des enseignants stagiaires: l'unité significative, le représentamen, les préoccupations et les connaissances.

\section{Lunité significative}

Elle désigne la plus petite structure significative du cours d'expérience de l'acteur. Elle peut être une action, une communication, un sentiment, une focalisation. À titre d'illustration, « regarde les élèves à l'atelier roulade avant et se dit qu'ils n'y arriveront pas » (sentiment- Karine, leçon 1).

\section{Les préoccupations}

Elles correspondent aux intérêts immanents à l'activité de l'enseignant stagiaire ici et maintenant en classe ou en formation « aider à la mise en place des trois ateliers »

\section{Le représentamen}

Cette composante correspond aux éléments qui sont perçus ou rappelés et qui font signes dans la situation pour éventuellement modifier l'action de l'enseignant stagiaire.

\section{Les connaissances}

Elles sont issues des expériences passées de l'enseignant stagiaire et correspondent aux éléments que celui-ci mobilise pour agir à l'instant t. Nous les distinguons en trois catégories: les connaissances mobilisées ou actualisées (les ateliers doivent démarrer en même temps pour que tous les élèves aient le même temps de pratique), les connaissances construites (pour que les élèves s'entraident, il faut que certains puissent rapidement devenir tuteurs), et les connaissances validées (contraindre le temps de recherche, limiter les débordements) ou invalidées par l'enseignant stagiaire (la fiche encourage les échanges entre élèves à propos de ce qu'il y a à faire.

L'exploitation du cadre d'analyse sémiologique et des différentes composantes retenues nous a permis de caractériser le développement professionnel des enseignants stagiaires. Celui-ci se donne à voir sous deux angles:
- d'une part au niveau de l'organisation propre de l'activité, notamment par la transformation et l'évolution de ses préoccupations et de ses différentes catégories de connaissances.

- d'autre part à travers l'analyse et l'observation comparée des préparations de leçon et de l'activité en classe, assurant ainsi une validité écologique aux analyses sémiologiques.

\section{Validité du traitement}

L'ensemble des étapes de cette analyse a fait l'objet d'une critique systématique des trois chercheurs coauteurs de l'article jusqu'à l'obtention d'un accord sur la documentation des composantes sémiologiques et le vocabulaire employé. Enfin la garantie de la validité du traitement des données a aussi été assurée dans un premier temps en soumettant aux enseignants stagiaires les transcriptions des données pour d'éventuelles modifications, puis en leur présentant les résultats.

\section{Résultats}

Dans le cadre de cet article, nous avons choisi de présenter une étude de cas (Yin 1994), afin d'éclairer de façon fine et circonstanciée le phénomène de développement professionnel. Les résultats retracent donc de façon synthétique et historique l'activité d'une enseignante stagiaire, Karine, qui a accepté d'être particulièrement disponible pour ce travail. Elle est intervenue auprès d'une classe de troisième en gymnastique pendant sept leçons de deux heures. Les données recueillies sur l'activité de Karine lors de la phase de préparation et lors de l'activité en classe, montrent la manière dont les connaissances construites dans et par l'action se transforment et deviennent prédominantes sur les connaissances théoriques pour agir et élaborer les plans d'action. Dans la perspective d'accroître l'efficacité du dispositif de formation, cette étude de cas longitudinale se prolonge par une analyse de l'activité collaborative de formation ${ }^{9}$ au sein du groupe de stagiaire. Les données recueillies sur Karine sont complétées par les verbalisations des autres stagiaires qui contribuent au développement professionnel de Karine. 


\section{D'une préparation conformiste à la construction d'un champ d'actions encouragées}

L'objectif initial que s'étaient fixé les stagiaires était de préparer des dispositifs d'enseignement en conformité avec une conception socio-constructiviste de l'apprentissage. Il s'agissait pour eux de trouver des réponses à la question suivante: comment planifier et que planifier pour une leçon pendant laquelle le processus d'apprentissage mobilisé par l'élève rejoint celui défini par une conception socio-constructiviste? Cette question qui apparaissait avant l'intervention en classe (puis après les analyses individuelle et collective de l'activité en classe pour les sessions 2 et 3) portait plus précisément sur la conception des tâches et dispositifs proposés aux élèves et sur ce que l'enseignant se prescrit à lui-même pour anticiper les mises en œuvre. Une difficulté récurrente évoquée par les stagiaires portait sur le choix de l'organisation pédagogique (mode de groupement, de communication, agencement des ateliers dans la salle) la mieux adaptée aux objets d'enseignement sélectionnés. À titre d'illustration, pour la leçon 3, Karine s'est posé la question suivante "quel aménagement de l'espace prévoir et quelles consignes donner pour favoriser l'acquisition par l'élève lui-même de connaissances et de compétences relatives à la liaison d'éléments gymniques appartenant à différentes familles? ». Dans le cadre de cet article, nous avons décrit et analysé la partie de la préparation de leçon en relation directe avec cet objet de notre étude.

\section{Une préparation conformiste}

L'objectif de cette préparation de leçon était de concevoir un dispositif d'enseignement pour que les élèves construisent des compétences spécifiques relatives à des éléments gymniques codifiés. C'est ainsi que l'enseignant stagiaire avait planifié une organisation en quatre ateliers: roulade avant, roulade arrière, appui tendu renversé, roue. Les élèves devaient être groupés par quatre ou cinq pour réaliser à tour de rôle l'élément gymnique. Pour chaque atelier, Karine avait réalisé un poster sur lequel était dessiné l'élément gymnique. On pouvait également observer la liste du matériel possible (tapis etc.) pour construire l'aménagement matériel ainsi qu'une présentation des critères de réussite. Le dispositif était complété par une fiche "élève » qui devait permettre de recueillir les réponses des élèves à la question « quels sont les critères de réalisation pour réussir cet élément?» (extrait préparation leçon 1). Pour Karine, les critères de réalisation sont les opérations (mouvements, postures, actions) qui doivent permettre à l'élève de réussir la tâche. L'organisation temporelle de la leçon prévoyait une rotation des élèves aux ateliers toutes les huit minutes auxquelles étaient ajoutées deux minutes d'échanges pour remplir la fiche. L'analyse de la préparation et des verbalisations de Karine, qui affichait une référence à la conception socio-constructiviste de l'apprentissage, a permis de pointer certaines contradictions. Tout d'abord la conception de la fiche est révélatrice d'une confusion entre la volonté délibérée de ne pas donner d'indications sur les opérations à réaliser et le fait d'inciter les élèves à échanger pour identifier ces opérations, et en conséquence de nouvelles compétences en gymnastique. Cet effet de masquage, en tant que pôle d'un dilemme dont l'autre élément serait le dévoilement, apparaît alors comme l'attribut archétypique de l'idéologie constructiviste, mais il n'aide pas les élèves à s'engager et transformer leurs actions. De leur point de vue de formateurs, les enseignants-chercheurs ont fait l'hypothèse que Karine n'a pas anticipé les difficultés des élèves liées aux larges possibilités d'interprétation dues à cette stratégie de masquage.

\section{Une préparation plus lucide}

À la suite de la première session de formation, une seconde préparation de leçon est réalisée. L'explicitation par Karine du plan d'action prévu a montré que des connaissances issues des expériences en classe ainsi que des conseils sous forme de règles professionnelles formulées lors du temps 3 de formation avaient été repris. Il s'agissait de construire un aménagement matériel susceptible de faire émerger un format pédagogique qui permette de concilier des préoccupations différentes et qui apparaissent parfois contradictoires: mettre rapidement les élèves au travail, contrôler les élèves et assurer l'engagement de chacun dans une activité collective coopérative pour apprendre, et enfin énoncer des consignes précises pour prescrire une tâche, pour organiser l'activité et assurer l'ordre. Karine a ainsi proposé des situations de résolutions de problème qu'elle a su argumenter tout en étant en mesure d'anticiper certains effets non désirés. En effet, elle précise qu'il n'est pas ques- 
tion de laisser trop d'initiative aux élèves concernant le contenu de l'échauffement, l'organisation et les thèmes des ateliers « là, je note toutes les actions que les élèves devront faire pour qu'ils soient rapidement en situation de travailler les enchaînements " (verbalisation préparation leçon 2). Karine a aussi retenu les conseils des enseignants-chercheurs et des autres stagiaires pour construire la tâche suivante: "dans trois espaces contraints, sous le regard d'observateurs, enchaîner quatre éléments gymniques en faisant le moins d'arrêts possible". "But: Dans chaque espace, enchaîner quatre éléments gymniques en faisant le moins d'arrêts possible. Un gymnaste / un observateur". Ce dispositif a été présenté par cette enseignante stagiaire comme en adéquation avec l'ambition de faire construire par les élèves le principe ou le critère de réalisation suivant: " pour une occupation optimale de l'espace et une limitation du nombre d'arrêts il est nécessaire d'opérer des changements de direction et de créer des éléments de liaison ». Pour susciter l'action des élèves, et des échanges favorables à la mise à distance de l'action, l'espace d'action à expérimenter délimitait une forme surprenante à l'aide de cinq tapis placés en croix. Karine avait rédigé les consignes précises à donner aux élèves: "Compétence visée: collaborer pour trouver l'ordre le mieux adapté pour enchaîner, sans marquer d'arrêts, une planche ou chandelle, une roue, une roulade avant, un appui tendu renversé (ATR) ou un trépied". L'observation de la préparation mettait en évidence la planification de nouveaux éléments. Karine a ainsi précisé qu'elle avait cherché à anticiper l'adaptation spontanée des élèves.

À titre d'exemple, en cas de difficultés persistantes, Karine avait prévu d'introduire progressivement des consignes sur les actions attendues (avoir plus d'amplitude etc.). L'énumération de quelques repères susceptibles d'aider les élèves à construire des procédures mieux adaptées aux contraintes proposées témoignait elle aussi d'une réelle évolution. Cependant Karine a précisé que certains conseils apparus pertinents en formation se sont avérés difficiles à exploiter dans le cadre de sa préparation de leçon: « j’ai préféré proposer une même configuration des tapis pour tous les groupes, je ne me sentais pas capable de proposer une configuration spécifique à chaque groupe ou de laisser chaque groupe choisir sa configuration » (verbalisation préparation leçon 2). À nouveau on perçoit une résistance face à la nécessité de prescrire: Karine semble s'excuser d'avoir imposé une organisation de l'es- pace, alors que la configuration imposée (tâche) est un élément susceptible de "poser problème » et de susciter une réorganisation de l'action.

\section{Une préparation fonctionnelle}

La dernière préparation que nous avons analysée s'appuyait sur la séquence de travail collectif (session 2 - temps 4). En effet Karine a retenu le plan d'action suivant: dans un espace contraint les élèves devaient enchaîner quatre éléments gymniques sans marquer d'arrêt. Trois aménagements matériels différents ont été conçus: le premier a consisté à disposer les tapis en forme de croix, le second en rectangle et le dernier en forme de « $\mathrm{L} »$. Les consignes prévues invitaient les élèves à déterminer l'ordre pour enchaîner une planche ou une chandelle, une roue, une roulade avant, un appui tendu renversé (ATR) ou un trépied: « là, j'ai noté qu'il fallait reprendre l'idée de présenter la tâche sous forme d'un défi entre groupes [connaissance validée lors de la leçon 2] » (verbalisation préparation 2). Selon sa conceptrice, ce dispositif devait inciter les élèves à expérimenter une variété d'actions, tout en étant guidé par les artefacts censés guider leur recherche de solution... L'objectif ici était de faire comprendre aux élèves que pour une occupation optimale de l'espace et une limitation du nombre d'arrêts il est nécessaire de faire des changements de direction et de créer des éléments de liaison. Dans une colonne intitulée "adaptations possibles", Karine avait noté les éléments de liaison à suggérer en fonction de telles ou telles difficultés.

Cette préparation de leçon montre une plus grande précision quant aux attentes de la stagiaire. Ce qui apparaît le plus planifié, tout en étant ouvert aux questions des élèves, ce sont les moments de présentation des consignes précédant la mise en activité et le questionnement relatif aux solutions proposées "là, j'ai passé beaucoup de temps pour trouver une forme d'organisation de mon discours pour capter et garder l'attention des élèves... C'est un peu une routine mais qui permet quand même de répondre aux questions des élèves » (verbalisation préparation leçon 3). En proposant trois aménagements spatiaux différents, Karine a explicité son intention de créer les conditions pour encourager une activité d'exploration individuelle et d'enquête pour résoudre les problèmes posés. À ce moment de la formation, 
la préparation semble envisagée par l'enseignante stagiaire comme une ressource pour l'intervention et non le plan de l'intervention. Autrement dit, pour la première fois, le dispositif est considéré comme un champ d'actions encouragées (Durand, 2008) où les actions sont validées ou non en fonction de leur pertinence par rapport aux contraintes fixées par l'enseignante stagiaire. Même si la préoccupation du masquage apparaît encore dans l'argumentation, elle n'en demeure pas moins sensiblement atténuée car elle ne s'impose plus comme un principe fondateur de sa conception constructiviste. En ce sens, on peut parler à ce moment d'une étape franchie dans le développement de l'enseignante stagiaire.

\section{Une activité en classe de plus en plus confortable et pertinente}

Dans le cadre de cette deuxième partie de résultat nous avons choisi de présenter à la fois les préoccupations les plus saillantes ayant organisé l'activité de Karine et les connaissances qu'elle a construites, mobilisées validées et invalidées lors des trois leçons.

Un aménagement matériel et une organisation des élèves inconfortables

À la fin de l'échauffement, Karine regroupe les élèves et leur annonce qu'ils vont travailler par ateliers, chacun d'eux étant consacré à un élément gymnique. Elle précise qu'avant de faire l'exercice, il est important de bien lire la fiche mise à disposition (cf. S1-T1). La période qui suit cette présentation est vécue par Karine comme inconfortable « là quand je les observe, je vois que certains sont déjà en train de pratiquer, d'autres font des roulades... c'est clair, ils lisent pas vraiment les fiches » (entretien leçon 1). Cette perception du contexte se traduit par une modification de l'engagement de Karine. Sa préoccupation dominante est alors de contrôler les élèves déjà en action et de mettre les autres au travail. Karine se lance alors dans un tour de salle pour observer les élèves et les interroger à propos de leurs actions « là, je passe dans les groupes pour rappeler les consignes et insister sur la sécurité... et je leur demande ce qu'ils font " (entretien leçon 1). Tout en s'organisant pour surveiller l'activité de la classe, l'intention de Karine est d'encourager les interactions entre les élèves de l'atelier roulade avant pour que chacun puisse réaliser une roulade avant simple ou écart sans utiliser les mains pour se relever: «là à ce moment, mon intention, c'est qu'ils échangent à propos de leur difficulté... j'attends que certains explicitent... comment ils se dégroupent; c'est pour cela que je propose à Karen d'observer Chloé » (entretien leçon 1). À l'atelier roulade arrière, Karine s'aperçoit que la fiche n'est pas vraiment une aide pour les élèves « je me rends compte que la fiche ne les intéresse pas, il y a sans doute trop d'informations, je me dis que la question 'quels sont les critères de réalisation ?'ça n'a pas beaucoup d'intérêt... surtout pour les élèves en difficulté ». L'enseignante stagiaire consacre la suite de son activité à répondre aux sollicitations des élèves qui sont en difficulté sur leur atelier: "je me rends compte que certains réussissent très vite et ensuite ils s'assoient pour discuter ou s'amuser $»$. Karine finit son tour de salle à l'atelier équilibre: « là, trois sur quatre n'y arrivent pas, ils sont vraiment en difficulté, alors je décide de leur proposer différentes solutions... sinon je sais qu'ils vont décrocher... et en plus ils peuvent se faire mal». Au cours de cette période, ce qui organise Karine, c'est d'assurer la continuité de l'activité des élèves dans les ateliers, tout en cherchant à mobiliser les plus inactifs. À plusieurs moments elle exprime son inconfort lié à la poursuite de préoccupations contradictoires: ne pas être prescriptive; ne pas perdre le contrôle des élèves; laisser plus de temps à certains groupes; réaliser la rotation des ateliers. Cet engagement en classe conduit Karine à construire des connaissances: " le niveau des élèves au sein de certains groupes ne leur permet pas d'échanger à propos du comment faire », " pour que les élèves s'entraident, il faut que certains puissent rapidement devenir tuteur », " pour l'atelier équilibre, ça peut être dangereux de ne pas interdire certaines actions » et invalide les connaissances « la conception de la fiche aide les élèves à faire des progrès », « la fiche encourage les échanges entre eux à propos de ce qu'il y a à faire ».

\section{Un dispositif prometteur}

Cette leçon est intervenue trois jours après les entretiens individuels et collectifs. La séquence analysée correspond à la mise en place et au fonctionnement de l'atelier consacré à l'enchaînement des 
éléments gymniques. Karine rassemble les élèves et leur explique qu'ils doivent réaliser 6 enchaînements (S2-T1) «vous travaillez les enchaînements indiqués au tableau puis vous discutez entre vous pour choisir l'enchainement le plus fluide... n'oubliez pas de remplir la fiche ». Là, je pense aux conseils de mon «conseiller péda ", j'essaie de bien construire mon discours, et de m'aider du tableau et des croquis... je veux vraiment qu'ils [les élèves] comprennent le pourquoi de cette proposition » (entretien leçon 2). Les élèves récupèrent une fiche de travail et s'affairent à construire l'aménagement matériel de leur atelier. Karine aide un premier groupe en leur indiquant où placer les tapis tout en interpellant un groupe de garçons qui s'amusent avec le chariot de tapis. Ses préoccupations sont « surveiller les élèves » et « les encourager à se mettre rapidement au travail $»$. Cinq minutes plus tard Karine intervient auprès d'un groupe de filles qui affirment avoir trouvé le bon enchaînement " d'accord, les filles... mais il faut essayer de le réaliser pour travailler le lien entre les éléments... je vous rappelle que tout arrêt est pénalisé ». Ensuite les préoccupations de Karine sont de « surveiller le travail des élèves tout en cherchant un groupe qui puisse valider l'efficacité du dispositif $»$. C'est en s'approchant de Kilian et Vincent qui discutent à propos de la difficulté à enchaîner roulade avant et roue, que ses attentes sont satisfaites « là, j'écoute et j'encourage Kilian à expliciter ce qu'il a perçu, ressenti lors de l'exécution des deux éléments...» (entretien leçon 2).

Au cours de cette séquence de vingt minutes, Karine valide des connaissances construites en (S1-T4), « contraindre le temps permet de limiter les débordements ", "présenter la tâche comme un défi facilite l'adhésion des élèves ", "les élèves recherchent s'impliquent davantage lorsqu'on leur donne des repères ", " l'activité des élèves est plus riche quand ils connaissent le motif de la tâche ", et construit de nouvelles connaissances « les élèves en réussite sont capables de dire ce qu'ils réalisent pour enchaîner les éléments », « un groupe composé d'élèves de niveaux différents et ayant des affinités favorise l'entraide et les échanges ».

\section{Un dispositif trop complexe}

Cette dernière leçon est consacrée à l'évaluation de l'enchaînement d'éléments gymniques dans les trois formats. Karine a affiché les trois posters présentant les trois configurations d'organisation des tapis avant d'entamer la présentation de la séquence. Les préoccupations de Karine sont de faire court et être le plus clair possible tout en s'assurant que les élèves aient compris. La suite est marquée par l'émergence de préoccupations concurrentielles: surveiller des élèves et guider d'autres dans leur recherche "Je voulais qu'ils cherchent un peu tout seul, qu'ils expérimentent... mais là je vois que ça ne marche pas pour David et Franck, ils essaient vite fait puis... et je vois aussi les autres qui sont tous regroupés à l'espace $3 »$. C'est aussi lors de cette séquence qu'elle a affirmé que la gestion des trois dispositifs était très inconfortable: "J'ai l'impression de ne pas avoir vu les élèves, j'étais sans cesse obligée d'intervenir pour les inciter à essayer d'autres combinaisons ... et à la fin là, je me dis que j'aurais dû imposer la place de certains éléments, notamment pour Céline et Fanny. Pour elles, il aurait été suffisant de trouver et construire les éléments de liaison dans deux dispositifs $»$. Un peu plus tard elle a insisté pour rappeler l'effet positif des fiches sur l'activité des élèves: "Les feuilles à remplir, c'est génial... ils ont vite compris qu’il fallait trouver la meilleure combinaison... et ils pouvaient aller voir la feuille d'un autre... et voir qu'il avait fait la même chose " et sa satisfaction de percevoir une forme d'émulation au sein de certains groupes « là, entre Marc et Benoît il y a compétition, c'est celui qui trouvera l'élément de liaison pour enchaîner avec le moins d'arrêts les éléments ». Les préoccupations évoluent et à cet instant Karine souhaite exploiter le travail du groupe de Marc pour encourager le groupe de Sylvie à explorer les différentes possibilités d'enchaînements.

Les connaissances construites par Karine sont: «l'organisation spatiale des tapis offre plus de possibilités pour engager les élèves dans une activité d'exploration », « les trois espaces ont permis aux élèves de comprendre que la solution est un ajustement singulier à un contexte particulier $»$. En outre elle valide les connaissances: " il ne suffit pas de mettre en place les ateliers pour que les élèves se transforment ", "l'identification et l'explication des opérations mises en ouvre pour réussir ne sont pas accessibles aux élèves les plus faibles » et invalide la connaissance: « le dispositif est adapté à tous les niveaux ». 


\section{D'une collaboration formelle orientée prescription à une collaboration fonctionnelle orientée dilemme}

Parmi les nombreuses préoccupations et attentes liées à la maîtrise de compétences professionnelles, c'est l'intention de mettre en ouvre une approche socioconstructiviste de l'apprentissage lors de l'enseignement de la gymnastique avec des élèves de troisième qui était à l'origine de la demande de Karine. Mais cette demande offrait un caractère particulier, celui d'être évoquée sous la forme d'une alternative apparemment insoluble: « je ne vois pas comment faire construire aux élèves leurs savoirs en gymnastique où tout est codifié... et puis quand je les laisse autonomes en atelier avec leurs fiches j'ai l'impression de plus rien maîtriser ». Plus généralement, les questionnements des autres enseignants stagiaires mettaient eux aussi en avant la contradiction apparente entre une conception de l'apprentissage où "l'élève a l'initiative ", et la difficulté à contrôler l'activité des groupes: " on sait pas combien de fois ils passent ", voire les échanges interpersonnels: "quand tu leur demandes de présenter leurs stratégies, t'en as toujours qui sont en train de jouer avec leurs raquettes en attendant que ça passe ", ou même de vérifier la fiabilité des relevés qui figurent sur les fiches d'observation: " des fois tu compares les fiches avec ce que les élèves font vraiment et là tu te demandes si on a vu le même film». La forme de cette demande, demeurait donc assez générique pour nous mettre dans la même incertitude que celle qui se présente aux chercheurs en ergonomie du travail enseignant (Amigues, Faita \& Saujat, 2004), à savoir que l'ambition formulée par les stagiaires ne permet pas aux enseignants-chercheurs de problématiser a priori les questions auxquelles ils sont réellement confrontés: attente par les stagiaires de modèles, de principes, de règles professionnelles, de recettes professionnelles... en vue de rechercher une plus grande conformité, une meilleure cohérence, un surplus de confort, un maximum d'efficacité...

La mise à l'épreuve des connaissances théoriques dans le guidage des stagiaires

Dans la mesure où tous les stagiaires nous ont fait part de remarques similaires concernant leur engagement professionnel, nous avons jugé nécessaire de mettre en perspective de la formation la construction progressive d'un « commun accord»: non seulement sur les notions de conception, de démarche d'enseignement et, ici plus particulièrement, de socio-constructivisme, mais aussi sur la place de ces conceptions et autres modèles théoriques de l'apprentissage dans l'engagement et le développement professionnel. C'est essentiellement en nous appuyant sur l'évolution des rapports entre l'action (mises en œuvre) et la conception (cadre d'analyse, technologie de planification...) que nous avons tenté de stabiliser celle-ci.

La conception « socioconstructiviste de l'apprentissage » présentée par Karine et les autres stagiaires véhiculait en effet des connaissances parcellaires, simplificatrices, et parfois erronées du fait de leur statut de doxa (Bourdieu, 1994). À ce titre ces connaissances relevaient d'avantage de croyances, de mythes, voire d'une éthique professionnelle que d'architectures théoriques ou génériques propres à guider ou orienter l'engagement professionnel. Ces connaissances fragilisées par leurs approximations dogmatiques (" les élèves doivent tout découvrir par eux-mêmes »), ont d'ailleurs pu engendrer des expériences professionnelles inconfortables, voire éthiquement irrecevables (« laisser faire les élèves en gymnastique peut être dangereux »). Au fil des échanges, c'est essentiellement la préoccupation d'une recherche de conformité par rapport aux attentes de certains de leurs formateurs qui a progressivement émergé. Karine a ainsi fait part d'une remarque d'un formateur, lors d'une visite, qui a participé à l'émergence de cette demande: " ta démarche d'enseignement est clairement annoncée socio-constructiviste mais tu dois quand même, à un moment donné, présenter clairement tes critères de réalisation pour que tes élèves soient autonomes dans la réussite de la tâche ».

Dans un premier temps, et devant ce type de préoccupation ancré sur une recherche de conformité au regard de ce qui apparaît comme une norme professionnelle (entrer dans la communauté de pratique des enseignants d'EPS), le rôle des enseignants-chercheurs a donc été essentiellement consacré à nuancer, relativiser la dimension prescriptive des connaissances scientifiques ou du moins expertes. Il s'est alors agi, non pas de les utiliser comme des règles pour légitimer l'appartenance à un genre professionnel (Clot, 1999), mais davantage de pointer leur intérêt pour interroger collectivement 
les mises en œuvre afin d'orienter indirectement et implicitement tant l'action que la réflexion sur l'action: («si, dans une conception constructiviste, apprendre c'est s'adapter à un environnement, comment aménager le milieu pour amener les élèves à réorganiser leurs habiletés motrices et leurs connaissances? »). Le questionnement s'est alors opéré par une circulation des préoccupations génériques (orientées conception) vers les préoccupations plus circonstanciées (orientées efficacité, voire expériences des élèves) et inversement. Cette démarche a permis de privilégier les interactions permettant aux uns et aux autres de s'inscrire dans une communauté de pratique de plus en plus libérée du point de vue surplombant et normatif de l'institution et de ses experts. Il est cependant apparu que certaines prescriptions s'avéraient nécessaires (et souhaitées) pour permettre au minimum aux stagiaires d'assurer sereinement le contrôle de la classe et de ne pas rester submergés par les urgences et les incertitudes: "là tu poses le problème trop tôt, ils sont pas encore tous dans l'action ", "quand tu as des fiches d'observations, assuretoi qu'elles sont bien comprises en comparant tes relevés à ceux des élèves, pour leur montrer que c'est important de pouvoir compter sur eux."

De la fertilité du conflit et des contradictions dans le développement professionnel

Dans un second temps, par un effet de balancier et sans doute à la faveur du dispositif d'auto-confrontation (temps 2) mis en parallèle du temps de formation collaboratif, ce sont les expériences de la conduite de la classe qui ont orienté la réflexion collective. Ces échanges ont été d'autant plus fertiles que certains stagiaires semblaient souffrir du décalage entre ce qu'ils se prescrivaient (cf. les fiches de préparation temps 1) pour faire apprendre les élèves (" proposer des SRP -situation de résolution de problème-») et leur activité réelle en classe ( « des fois, on est quand même obligé de donner des réponses »). Pour Karine, si bien enseigner consiste encore à « concevoir des tâches et guider les apprentissages en référence à une théorie précise de l'apprentissage », il apparaît néanmoins que les limites de sa conception sont désormais tracées par son expérience des nécessités pratiques: "là, je leur montre comment, en fermant vite le bassin ils accélèrent, sinon ils vont se décourager ». Ce type de réaction entre pairs devant la vidéo témoigne d'une prise de distance entre ce que chacun se prescrit devant son ordinateur, au bureau, et les expériences contrastées de la mise en œuvre. On retrouve ces remarques, plutôt circonstanciées lors de cette étape, lors des échanges avec les autres stagiaires: "moi, en hand, quand ça tourne en rond, je leur impose une tactique défensive ", "en triple t'es souvent obligé de leur aménager des espaces avec des lattes ${ }^{10}$, sinon ils sentent rien ", "j'ai remarqué avec mes sixièmes qu'il y a trop de différences entre eux, alors, en fait je leur laisse pas trop le temps de chercher». Ces échanges élargis ont porté sur de nombreux thèmes comme les modes de groupement ("Moi, j'ai envie de te dire qu'il faut les faire travailler par groupe de niveau pour qu'ils cherchent collectivement »), ou sur la dynamique du groupe («On pourrait proposer un système de marque où chacun pourrait marquer des points pour l'équipe, moi je l'ai fait en lutte... ça marchait très bien. Si tu veux je pourrai t'expliquer comment j'ai fait». Ces discussions ont été guidées par le souci des enseignants-chercheurs d'inviter les stagiaires à envisager et discuter d'autres choix pour les leçons à venir en abordant les notions de saisie d'opportunités et d'ouverture des possibles hic et nunc. Le pas en avant dans le développement professionnel consiste ici à prendre le risque de se libérer, lorsque cela apparaît opportun, des prescriptions et du cadrage de la fiche de préparation.

Toujours en se référant au cadre conceptuel socioconstructiviste, les temps de partage d'expériences ont permis de cibler les préoccupations sur l'activité réelle des élèves et des stagiaires. Les anciennes croyances ont été tordues (" tu es sûr que, présentée comme ça, ta fiche d'observation peut servir de support à la confrontation? »), déformées ("tu penses pas qu’à ce moment c'est plutôt les conditions de production de la

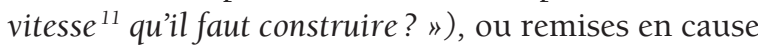
(" pourquoi tu ne lances pas un défi sur le temps de renversement plutôt que de leur demander comment ils ont réussi? "). Pour autant, la préoccupation initiale de s'engager dans une conception socio-constructiviste de l'apprentissage et de l'enseignement n'en est pas moins restée une " boussole » commune pour interroger à la fois l'efficacité, le réalisme, le confort et la cohérence des mises en ouvre.

\section{Discussion}

Cette recherche/intervention avait pour ambition de répondre aux préoccupations ambitieuses des 
enseignants stagiaires autour de la demande d'enseigner en référence à une conception socio-constructiviste. Pour répondre à cette force d'appel (Schwartz, 1997) et relever ce défi, des temps de formation ont systématiquement alterné des approches couplées: analyse de la demande/positionnement conceptuel, positionnement conceptuel/planification des dispositifs d'enseignement, mise en œuvre/validation des dispositifs. Cette formation a permis aux stagiaires de s'engager dans une perspective de développement professionnel et d'appréhender concrètement l'engagement dans des opérations caractéristiques du métier d'enseignant telles que: « concevoir et mettre en œuvre son enseignement, travailler en équipe, se former et innover » (référentiel de compétences des IUFM). L'alternance des différentes phases a aussi permis aux enseignants stagiaires de faire, de manière circulaire, trois types de liens: 1 ) des liens entre les contraintes dilemmatiques de leur mission (travailler dans la sérénité et le " confort » tout en ouvrant la « marge de manœuvre » des élèves), 2) des liens entre les différentes expériences de leurs pairs et leur vécu propre (les expériences des uns et des autres, fussent-elles malheureuses, devenant des ressources pour tous), 3) des liens entre des circonstances singulières à affronter et des conceptions aux dimensions plus génériques. Nous avons cherché à orienter et à analyser notre action de formation en nous préoccupant du développement professionnel des enseignants stagiaires par des processus de validation/ invalidation impliquant la mobilisation de référentiels multiples et complémentaires: effets attendus chez les élèves, sensation de confort « ergonomique », écart à la conception socio-constructiviste annoncée, écart aux dispositifs planifiés. La mobilisation de cette activité d'analyse multi-référencée a permis d'observer comment les stagiaires parviennent à déstabiliser tant leurs connaissances théoriques relatives à la conception socio-constructiviste (générique/circonstancié) que les gestes professionnels relatifs à l'intervention (planification/improvisation), pour stabiliser de nouvelles connaissances indissociables de l'action et pour l'action. Dans le cadre de cet article, nous proposons de discuter quatre points: la circularité des connaissances comme processus du développement professionnel, l'effet transformateur des entretiens individuels et collectifs, la migration/ circulation des connaissances comme saisies d'opportunités, l'évolution des préoccupations co-construites lors des séquences de formation.

\section{La circularité des connaissances comme processus de développement professionnel}

Les résultats ont montré que le développement des enseignants stagiaires était dynamique, complexe et intervenait lors des différents temps de formation. Les conditions de l'alternance entre les temps de formation à l'IUFM et les expériences professionnelles en classe ont ainsi permis aux stagiaires d'acquérir des connaissances en phase à la fois avec leur demande initiale (assumer une conception socio-constructiviste) et avec les préoccupations qui ont émergé lors des différents temps de leur formation (être plus efficace, mieux vivre les situations $a$ priori anxiogènes d'urgence et de dilemmes...). Les échanges initiaux ont montré que le modèle initial de mobilisation des connaissances privilégié par les stagiaires était indexé à une approche déductive, technologique et rationnelle du métier. Celle-ci amenait les enseignants stagiaires à penser selon une causalité mécanique, à la fois restreinte et suffisante (de leur point de vue) entre la réussite de l'intervention en classe et les modalités de rationalisation qui l'ont préalablement étayée. Cette dimension du métier d'enseignant, que constitue la planification, est en effet souvent appréhendée par les débutants comme le moment d'une réflexion qui doit s'affranchir des contingences de l'action pour se développer, voire s'épurer dans l'espace et le temps interlopes, à la fois professionnels et privés, que constitue le bureau de l'enseignant. La formation par alternance a permis collectivement aux stagiaires de valider ou d'invalider certaines des connaissances concernant la fonction de la préparation de la leçon et ses modalités. En effet, nous avons montré que le travail de rationalisation des situations d'apprentissage fondé sur le souci de réduction des décalages entre tâches prescrites et conception de l'apprentissage a été rapidement dépassé pour s'engager dans la simulation de scénarii. Les enseignants stagiaires ont alors appris que la réussite de leur intervention ne dépendait pas seulement de la qualité de la rationalisation mais de leur capacité à interroger l'activité réelle des élèves ainsi que le rapport action/plan qui est inversé lors de cette recherche/intervention.

Le gain d'efficacité en classe a ainsi été obtenu en partie par l'attribution de fonctions nouvelles aux préparations de leçons. En effet, progressivement nous avons montré qu'après avoir cherché à 
rendre la préparation conforme à une conception de l'apprentissage, ou à une demande d'un formateur, l'enseignant stagiaire a construit un plan d'action permettant davantage de concilier des préoccupations concurrentes, voire discordantes. Les résultats ont montré que le modèle de mobilisation des connaissances était devenu plus sensible à une approche inductive, pragmatique et fonctionnelle du métier permettant de construire une voie moyenne entre les choses de la logique et la logique des choses (Bourdieu, 1980).

\section{L'effet transformateur des entretiens individuels et collectifs}

Parmi les moments propices au développement professionnel, les sessions consacrées aux entretiens individuels puis aux débats collectifs semblent avoir eu un effet positif sur la validation ou l'invalidation de connaissances relatives à la conception, la présentation et la régulation de situations pédagogiques. Les stagiaires ont en effet exploité pleinement la possibilité de revoir les traces de leur activité en classe pour comprendre certains dysfonctionnements, et envisager des actions alternatives à ce qu'ils avaient réalisé et / ou prévu. Lors de ces instants, l'entretien, qui est une activité sur l'activité passée (Riff, Pérez, Grison, \& Guérin, 2000), a des effets transformateurs déterminants. En effet, en dehors de toute pression temporelle, l'enseignant peut prendre le temps de visionner plusieurs fois les séquences afin de comprendre telles ou telles situations. C'est ainsi qu'il peut percevoir de nouveaux éléments jusqu'à présent non remarqués, des éléments qui donnent lieu à une interprétation différente du contexte et à la validation/invalidation de connaissances. Ce phénomène identifié par Riff et al. (2000) a été analysé par Sève \& Adé (2003) dans le cadre de l'étude de l'activité d'un enseignant lors d'entretiens d'autoconfrontation. Les auteurs ont montré l'existence de chaînes interprétatives débordant celles réalisées au cours de la leçon et donnant lieu à la création de nouvelles connaissances ou à l'invalidation de connaissances censées être utiles. Si notre étude a permis de repérer le même phénomène, on note cependant une différence liée à la dimension collective de l'entretien. En s'appuyant sur leur propre expérience professionnelle, les stagiaires à différents niveaux ont participé au développement de longues chaînes interprétatives. Les nouvelles interprétations de l'activité passée s'accompagnent de la construction de connaissances pour l'action, des connaissances qui offrent des perspectives nouvelles pour agir dans des situations présentant un air de famille avec celles qui ont été étudiées. À un autre niveau, ce lieu d'échanges social entre formateurs et enseignants stagiaires nous semble propice pour construire une mémoire collective des manières d'agir et d'astuces pour intervenir en classe. En suivant Saujat (2004), on peut dire que les expériences des uns et des autres contribuent à stabiliser des façons de faire le métier, des formes d'actions types qui permettent de dire qu'un "genre professionnel des enseignants débutants " se construit progressivement. En participant à cette recherche/ intervention, on peut dire que l'enseignant construit non seulement des connaissances issues de la compréhension de sa propre expérience mais surtout d'une mémoire collective (des systèmes ouverts de façons de faire et de penser partagées), véritable ressource pour agir et se développer. L'analyse de l'activité des enseignants débutants a montré que ces ressources étaient davantage une ouverture de leur horizon expérientiel (Theureau, 2004), qu'un gain immédiat d'efficacité en classe.

\section{La migration des connaissances comme saisies d'opportunités}

Comme dans tout dispositif de formation, la pertinence des connaissances construites par les stagiaires ainsi que leur opérationnalité est très difficile à mesurer, car les opportunités de les mobiliser sont aléatoires et particulièrement circonstanciées. En effet, même si certaines règles trouvent suffisamment d'événements récurrents pour être validées ou non, d'autres connaissances construites lors de la session 1 ou 2 ne seront mobilisées lors de l'action en classe que si les circonstances s'y prêtent. Les enseignants stagiaires soulignent d'ailleurs à plusieurs reprises la difficulté lors de l'intervention en classe d'exploiter certains conseils jugés pertinents. Certains auteurs confirment ce constat en rappelant que ce qui est appris en situation d'entretien n'est pas nécessairement source de transformation en classe et que les connaissances ne migrent pas mécaniquement d'un lieu à un autre, ni de l'analyse de l'action vers l'action elle-même, ni de l'expérience vécue vers sa conceptualisation. Ce 
phénomène dit « d'adhérence » mis en évidence par Serres et al. (2004) chez des enseignants stagiaires en EPS semble être récurrent. Néanmoins, les décalages observés entre l'action prévue en formation (ce que se prescrit l'enseignant stagiaire) et l'action réalisée en classe (ce qu'il fait) ne signifient pas qu'il n'y a pas de développement professionnel. Si les progrès ne se manifestent pas immédiatement on peut quand même penser qu'envisager de nouveaux possibles, notamment en construisant des scenarii plausibles pour l'action en classe, est une forme de développement des stagiaires. Pour accroître les chances de migration des connaissances et des règles du métier du lieu de leur formalisation vers la classe, Méard \& Bruno (2009) proposent plusieurs démarches d'intervention des formateurs basées sur la prescription. En effet, selon les auteurs, le rôle du formateur lors des phases de conseils ou de tutorat n'est pas de suivre à la lettre une démarche socio-constructiviste ou le savoir enseigné serait systématiquement masqué. Pour Méard et al. (2009), c'est surtout en établissant des liens entre des éléments d'expérience ${ }^{12}$ et la règle professionnelle à construire que la difficulté à opérer une migration fonctionnelle (Clot, 2003) de la règle dans d'autres contextes pour des usages similaires peut être en partie contournée. Adoptant cette approche de la formation, Chaliès, Flavier, \& Bertone (2006) ont mis en évidence, lors de l'analyse d'une situation innovante de conseil pédagogique que lors de la co-intervention avec son conseiller pédagogique en classe, le stagiaire mobilisait les règles de métier apprises lors de la co-préparation de la leçon. La fécondité de cette démarche de formation semble confirmée par nos résultats. En effet, à plusieurs reprises le formateur est intervenu pour présenter la règle à construire tout en pointant les éléments à considérer pour en faciliter son acquisition par les stagiaires afin d'élargir leur pouvoir d'action en classe. Au cours des temps de formation à l'IUFM, nous avons ainsi pointé les liens de signification entre les différentes règles portant sur le « quoi faire » puis énoncé le « comment faire » pour co-construire les opérations à réaliser. Pourtant selon le présupposé d'autonomie de l'activité humaine, il est important de rappeler que ce sont les formés euxmêmes qui «ont la main » sur leur propre activité (ce qui a été formulé en termes d'auto-organisation). La prescription du formateur ne commande pas l'activité de l'enseignant stagiaire; elle reste un élément parmi d'autres qui, en fonction de l'interprétation du contexte, pourra devenir une ressource pour agir.
Évolution des préoccupations co-construites lors des séquences de formation

Par le biais du dispositif proposé, les stagiaires ont construit une posture pragmatique concernant la conception socio-constructiviste de l'apprentissage. À travers les sessions de formation en dehors de la classe, ceux-ci ont progressivement abandonné le projet illusoire d'appliquer stricto sensu une conception de l'apprentissage socio-constructiviste. Ils ont adopté une posture plus pragmatique où les savoirs théoriques ne sont que des ressources parmi d'autres pour agir de manière efficace et cohérente au regard de préoccupations tant « économiques » qu'axiologiques. C'est grâce notamment à l'analyse de séquences d'activité en classe jugées comme efficaces que la perspective " déductive » d'utilisation des savoirs théoriques a été abandonnée. Ces résultats semblent confirmer le constat fait par Saujat (2001) lors d'un épisode d'instruction au sosie menée avec une stagiaire en éducation physique. L'auteur souligne que le sens attribué à la signification d'un modèle théorique d'apprentissage diffère entre le cadre d'un travail décontextualisé en formation et celui d'une expérience quotidienne d'enseignement. (Saujat, 2004).

Ce nouveau positionnement par rapport à la théorie socio-constructiviste a permis aux stagiaires de se positionner de manière critique par rapport à un modèle à partir duquel ils ont compris que tout vagabondage est possible. Toutefois, ce bricolage heuristique exige que les enseignants stagiaires aient construit une "boussole pédagogique" leur permettant de s'engager profitablement dans le dilemme auto-conservation/auto-transformation. On pourra ici parler d'un glissement où le modèle théorique a vu sa fonction transformée, passant d'une ressource discursive (comprendre et accéder au domaine des connaissances sur les apprentissages) en une ressource praxique (agir dans la classe de manière confortable, efficace et cohérente). Les enseignants stagiaires ont compris que, face à la complexité du métier d'enseigner, la réussite de l'intervention en classe passait par la mobilisation de connaissances plurielles et que celles-ci pouvaient être contradictoires entre elles. On peut considérer que cette forme de développement professionnel conquis en dehors de la classe s'est aussi donnée à voir par la primauté accordée à la compréhension de l'activité réelle des élèves. À la suite de Méard et Bruno (2009), on 
peut caractériser ce pas en avant, c'est-à-dire cette nouvelle façon d'envisager l'action en classe, comme la subordination des prescriptions descendantes (préparation de leçon, programmes etc.) aux prescriptions ascendantes (ce que font les élèves).

\section{Conclusion}

Les premiers pas dans le métier d'enseignant sont le plus souvent accomplis en cherchant à mettre les élèves au travail, maintenir l'ordre, " avoir une classe qui tourne », à résoudre des dilemmes (Ria, Saury, Sève, \& Durand, 2001) plutôt qu'avec l'ambition de faire apprendre en se conformant à une conception de l'apprentissage (Durand, 1996). En nous demandant d'accompagner leurs premiers pas autour d'une exigence de cohérence théorique - pratique, les enseignants stagiaires nous ont engagés sur une perspective qui pouvait relever du défi. Nos résultats montrent cependant qu'à certaines conditions, les enseignants stagiaires peuvent, dans le cadre d'une formation par alternance, se développer professionnellement en poursuivant simultanément les trois préoccupations ambitieuses que sont la recherche de confort, le souci de l'efficacité, le respect des principes axiologiques d'une conception de l'apprentissage. De manière spéculative et prospective il semblerait que ce type de dispositif puisse offrir des pistes de réflexions intéressantes pour penser la future formation des enseignants d'EPS en l'adaptant aux nouvelles exigences d'un métier qui, invite (charte des IUFM) et incite (article 34 de la loi d'Orientation et de Programme pour l'Avenir de l'École 2005) à l'innovation, à la nécessité de comprendre, de conceptualiser et de situer ses pratiques et celles des élèves, notamment à l'aide des «boussoles pédagogiques » construites en formation.

Si la posture de formateur des enseignants chercheurs dans le dispositif de formation assurait une action de formation respectueuse de l'épistémologie de l'action, l'engagement qui consistait conjointement à étudier l'activité des enseignants stagiaires demande effectivement une certaine capacité à dissocier ces postures. C'est pourquoi, dans nos travaux en cours étudiant l'activité individuelle-collective des formés et du formateur, nous avons choisi de privilégier des dispositifs où nous n'étions pas directement impliqués dans le temps de formation. On se rapproche ainsi de la démarche itérative alternant temps de recherche et temps de formation, instituée dans l'ingénierie de formation des enseignants proposé par Ria et coll (2006). 


\section{NOTES}

1. Cette entrée par l'expérience signifie que le dispositif de formation est orienté prioritairement par l'activité des stagiaires en classe plutôt que sur la base de savoirs à maîtriser.

2. Nous revendiquons cette orientation plutôt que celle de recherche action afin de caractériser la double visée de la démarche: décrire et comprendre l'activité de l'enseignant stagiaire en situation de classe et, à partir de l'analyse de l'activité, aider en formation à la conception de nouvelles situations d'enseignement.

3. Un enseignant stagiaire est une personne titulaire d'une licence $(b a c+3)$ ayant réussi le concours de recrutement (CAPEPS: Certificat d'Aptitude Professionnelle à l'Enseignement de l'Éducation Physique et Sportive).

4. Les représentations et les connaissances des enseignants stagiaires relatives à cette conception ont été appréhendées à partir d'un questionnaire distribué en début de formation.

5. Il a été précisé notamment que les enseignants stagiaires pouvaient à tout moment interrompre sans justification leur participation.

6. Il s'agissait de gommer les effets « pervers » liés à la posture de formateur/évaluateur.

7. C'est-à-dire des règles qui peuvent s'appliquer à un grand nombre de situations, voire toutes les situations du métier, par exemple: il faut relancer l'attention des élèves.

8. C'est-à-dire des règles incarnées dans un petit nombre de situations, voire une seule situation, dans des circonstances particulières, par exemple: à ce moment, avec cette classe, il faut regrouper et faire asseoir les élèves à tel endroit et de telle façon.

9. Selon l'épistémologie de l'expérience (entrée activité) concevoir ou améliorer des dispositifs de formation passe par une analyse de l'activité en action et en formation. Pour une présentation détaillée se reporter à Durand (2008).

10. Lors de situations de triple saut, les enseignants placent fréquemment des lattes sur le sol pour délimiter des zones afin de faciliter la perception de certains éléments externes, et, ou, internes et de favoriser leurs mises en relations.

11. La figure gymnique évoquée est l'appui tendu renversé.

12. Pour une présentation complète des démarches de construction des règles professionnelles, se reporter à Méard \& Bruno (2009). 


\section{RÉFÉRENCES}

Altet, M. (2000). L'analyse de pratiques, une démarche de formation professionnalisante? Recherche et Formation, 35, 25-41.

Amigues, R., Faìta, D., \& Saujat, F. (2004). « L'autoconfrontation croisée » : une méthode pour analyser l'activité enseignante et susciter le développement de l'expérience professionnelle. Bulletin de Psychologie, 469, $41-44$.

Anderson, D.J. (1992). Teacher supervision that works: A guide formation university supervisors. Greenwood Publishing Group.

Barbier, J.M. \& Durand, M. (2003). L'activité: un objet intégrateur pour les sciences sociales? Recherche et Formation, 42, 99-117.

Bertone, S., Chaliès, S., Clarke, A., \& Méard, J.A., (2006). The dynamics of interaction during post-lesson conferences and the development of professional activity: Study of a preservice physical education teacher and her cooperating teacher, Asia Pacific Journal of Teacher Education, 34(2), 245-264.

Bourdieu, P. (1980). Le sens pratique. Paris: Éditions de minuit.

Bourdieu, P. (1994). Raisons pratiques. Paris: Seuil.

Brusque, L. \& Henry, Y. (1998). Une étude critique d'un modèle de formation conjointe et ses limites. In D. Raymond et Y. Lenoir (Eds.), Enseignants de métier et formation initiale (pp. 175-194). Bruxelles: De Boek Université.

Cano, F. \& Cardelle-Elawar, M. (2004). An integrated analysis of secondary school students' conceptions and beliefs about learning. European Journal of Psychology of Education, 19 (2), 167-187

Chaliès, S. \& Durand, M. (2000). Note de synthèse: L'utilité discutée du tutorat en formation initiale des enseignants. Recherche et Formation, 35, 145-180.

Chaliès, S. Flavier, E., \& Bertone, S. (2006). Vers une rénovation de la situation traditionnelle de conseil pédagogique. Communication présentée à $4^{\circ}$ biennale de l'ARIS « Co-construction des savoirs ». Besançon. France. Mai.

Clot, Y. (1999). La fonction psychologique du travail. Paris: PUF.

Clot, Y. (2003). Le collectif dans l'individu? in G. Vallery et R. Amalberti (Eds.), Modèles et pratiques de l'analyse du travail 1988-2003, 15 ans d'évolution. Actes du $28^{\circ}$ Congrès de la SELF. Paris: SELF.

Clot, Y. (2007). De l'analyse des pratiques au développement des métiers. Revue Éducation \& Didactique, 1, 83-94.

Clot, Y. Faïta, D. Fernandez, \& G. Scheller, L. (2001). Entretiens en autoconfrontation croisée. Une méthode en clinique de l'activité. Éducation Permanente, 146, 25-37.

Cros, F. (1999). Le mémoire professionnel en formation d'enseignants. Paris: L'Harmattan.

Daniellou, F. (1996). L'ergonomie en quête de ses principes. Toulouse: Octarès.

Durand, M. (2008). Un programme de recherche technologique en formation des adultes: une approche enactive de l'activité humaine et l'accompagnement de son apprentissage/développement. Revue Éducation E Didactique, 3(2), 69-93.

Durand, M. (1996). L'enseignement en milieu scolaire. Paris: PUF.

Durand, M., de Saint Georges, I., \& Meuwly-Bonte, M. (2006). Le curriculum en formation des adultes: Argumentation pour une approche « orientée-activité ». Raisons Éducatives, 10, 185-202.

Gonnin-Bolo, A. (2002). Le mémoire professionnel en , "traduction des savoirs, "médiation" des formateurs?" Recherche et formation, 40, 59-74. 
Leblanc, S., Ria, L., Dieumegard, G., Serres, G., \& Durand, M. (2008). Concevoir des dispositifs de formation professionnelle des enseignants à partir de l'analyse de l'activité dans une approche enactive, @ctivités, 5 (1), 58-78.

Méard, J. (2004). L'analyse de pratiques au quotidien. Éducation Permanente 161, 45-53.

Méard, J. \& Bruno, F. (2009). Les règles du métier dans la formation des enseignants débutants. Toulouse: Éditions Octarès.

Mialaret, G. (1996). Savoirs théoriques, savoirs scientifiques et savoirs d'action en éducation, in Barbier, J-M. (Ed.), Savoirs théoriques et savoirs d'action (pp. 161-187). Paris: PUF.

Ria, L. (2006). L'entrée dans le métier des enseignants du second degré: un programme de recherche centré sur l'analyse de l'activité, Habilitation à Diriger les Recherches, Clermont Ferrand: Université Blaise Pascal.

Ria, L., Leblanc, S., Serres, G., \& Durand, M. (2006). Recherche et formation en « analyse de pratiques ", Recherche et Formation, 51, 43-56.

Ria, L., Saury, J., Sève, C., \& Durand, M. (2001). Les dilemmes des enseignants débutants. Études lors des premières expériences de classe en éducation physique. Science et motricité, 42, 47-58.

Riff, J. Pérez, S., Grison, B., \& Guérin, J. (2000). De l'autoconfrontation comme méthode à l'entretien en situation d'autoconfrontation comme activité située: points de discussion et propositions méthodologiques. $2^{\text {nde }}$ journée de travail «modélisation de l'expérience individuelle et collective », 12 mai, Paris.

Saujat, F. (2001). Quand un professeur des écoles débutant instruit son "sosie" de son expérience. Les Dossiers des Sciences de l'Éducation, 7, 107-117.

Saujat, F. (2004). Spécificités de l'activité d'enseignants débutants et "genres de l'activité professorale". Polifonia, $8,67-93$.

Saujat, F., Amigues, R., \& Faïta, D. (2005). Les compétences pour enseigner comme ressources et produits de l'activité du professeur, in Talbot, L., et Bru, M. (Eds.), Des compétences pour enseigner(pp. 183-197). Rennes: Presses Universitaires de Rennes.

Schwartz, Y. (éd.) (1997). Reconnaissance du travail, pour une approche ergologique. Paris: PUF.

Serres, G., Ria, L., \& Adé, D. (2004). Modalités de développement de l'activité professionnelle au gré des contextes de classe et de formation: le cas des professeurs stagiaires en Éducation Physique et Sportive, Revue Française de Pédagogie, 149, 49-64.

Sève, C. \& Adé, D. (2003). Les effets transformatifs d'un entretien d'autoconfrontation: une étude de cas avec un enseignant stagiaire. $5^{\circ}$ journées d'étude Act'Ing « l'observatoire des objets d'analyse de l'activité humaine », St Pierre de Quiberon, 30 au 30 mai.

Tardif, M \& Lessard, C. (1999). Le travail enseignant au quotidien. Expérience, Interactions humaines et dilemmes professionnels. Bruxelles: De Boeck.

Theureau, J. (2004). Cours d'action: méthode élémentaire. Toulouse: Octarès Éditions.

Theureau, J. (2006). Cours d'action: méthode développée. Toulouse: Octarès Éditions.

Wittgenstein, L. (2004). De la certitude. Paris: Gallimard.

Vygotski, L.S. (1997). Pensée et langage. Paris: La Dispute.

Wenger, E. (1998). Communities of pratice. Learnin, Meaning and Identity. Cambridge: Cambridge University Press.

Yin, R. K. (1994). Case study research: Design and methods ( $2^{\text {nd }}$ ed.). Beverly Hills, CA: Sage Publications. 